\title{
SiETE CONTROVERSIAS CAPITALES: ANÁLISIS DE LA IMPLEMENTACIÓN del Programa Maíz Solidario en Los Altos de Chiapas
}

\section{Seven Capital Controversies: Analysis of "Maíz Solidario" Program in Los Altos de Chiapas}

\author{
Renzo D'Alessandro-Nogueira \\ Alma Amalia González-Cabañas
}

\begin{abstract}
Resumen: El objetivo de este artículo es entender con precisión las contradicciones, acuerdos y retos de los modelos de conservación de la biodiversidad de maíces, a través del análisis del paradigma tecnológico dentro del Programa Maíz Solidario aplicado en Los Altos de Chiapas. Con base en conceptos y la metodología de la sociología de la traducción, se consideró la realización de un panel con actores involucrados en la aplicación del Programa, cuyos discursos se agruparon en siete controversias. Entre las conclusiones se valora la noción de red sociotécnica para dicho análisis y la necesidad de construir acuerdos epistemológicos al interior de esta red.
\end{abstract}

Palabras clave: conservación, biodiversidad, maíces nativos, red sociotécnica, paradigma tecnológico.

Abstract: The aim of this paper is to understand more accurately the contradictions, agreements and challenges of the conservation models of maize biodiversity through the analysis of the technological paradigm applied in the Programa MaizSolidario in Los Altos de Chiapas. Using concepts from the Actor-Network-Theory, we used a focus group with the stakeholders involved in the Program's implementation whose speeches were grouped in seven controversies. The main conclusions value the notion of sociotechnical network for this kind of analysis and the need to establish epistemological agreements into the network.

Keywords: conservation, biodiversity, corn landraces, socio-technical network, technology paradigm.

Renzo D’Alessandro Nogueira, doctorante en socio-antropología en la Universidad Sup Agro, Montpellier III, UMR: Innovation, Francia. Investigador huésped del Programa de Investigaciones Multidisciplinarias sobre Mesoamérica y el Sureste, de la Universidad Nacional Autónoma de México (PROIMMSE-UNAM), en San Cristóbal de Las Casas, Chiapas, México. Temas de especialización: evolución de sistemas agrarios, economía campesina, desarrollo rural, procesos de innovación científico-tecnológicos, conservación de maíces nativos, estructuras tradicionales comunitarias y acción colectiva de los pueblos indígenas. Correo electrónico: renzo.dalessandro@gmail.com.

Alma Amalia González Cabañas, investigadora titular A del Programa de Investigaciones Multidisciplinarias sobre Mesoamérica y el Sureste, de la Universidad Nacional Autónoma de México (PROIMMSE-UNAM), en San Cristóbal de Las Casas, Chiapas, México. Temas de especialización: problemas del medio rural, desafíos de la agricultura campesina ante el sistema agroalimentario global, comercio justo, agricultura orgánica de organizaciones campesinas, seguridad alimentaria, mercados alternativos locales y hábitos alimentarios en pueblos indígenas. Correo electrónico: agonzale@unam.mx; alma.amalia@gmail.com.
Enviado a dictamen: 04 de octubre de 2013. Aprobación: 17 de diciembre de 2013. Revisiones: 1. 


\section{A manera de introducción: dos modelos de conservación}

$\mathrm{M}$ éxico es un país megadiverso en cuanto a especies animales y vegetales se refiere. Se calcula que en todo el país se utilizan más de veintiséis mil especies de plantas (Groombridge y Jenkins, 2002), cinco mil plantas útiles (Mariaca et al., 2007) y 237 variedades de maíz de 59 razas nativas ${ }^{1}$ desarrolladas desde hace cerca de ocho mil años, por 350 generaciones de agricultores (Boege, 2008). La diversidad de maíces nativos cumple diferentes funciones: sociales, al aportar la base alimentaria de la sociedad; económicas, al asegurar una actividad productiva para la población campesina; culturales, en cuanto a que intervienen factores rituales, culinarios y mitológicos ligados a su producción; y genéticas, al ser adaptables a los diferentes suelos y climas existentes en el país, cuyos rendimientos y resistencia a plagas o enfermedades varían según la raza y la variedad. Estas diferentes funciones que cumplen los maíces nativos hacen de su conservación una cuestión prioritaria para el Estado y para la sociedad mexicana en su conjunto.

Identificamos conceptualmente dos modelos de conservación de la biodiversidad cultivable: uno biotecnológico y otro agrícola-tradicional. El modelo biotecnológico supone un cambio estructural en el papel que juegan las sociedades campesinas, que han pasado de ser los desarrolladores naturales de la biodiversidad a simples usuarios de tecnología genética provista por las empresas de biotecnología. El mercado y sus leyes se posicionan como garantes de la preservación de la biodiversidad de plantas cultivadas. La conservación es entendida como el resguardo de los recursos genéticos, ya sea in situ, mediante complejas técnicas de conservación de germoplasma en un modelo que llamamos "agrícola tradicional", o ex situ, mediante campos de reproducción de semillas y bancos de germoplasma. ${ }^{2}$ Este segundo modelo exige un marco jurídico formal en el que, con la ayuda gradual del Estado, los campesinos podrán convertirse en "fitomejoradores" y proveedores de semillas con derechos de propiedad intelectual (Aguirre, 2011).

En el modelo "agrícola-tradicional", en cambio, se concibe la conservación como un proceso de reproducción social y cultural asociado al ciclo biológico de la planta y a la reproducción social de la organización campesina. Los conocimientos con él generados proporcionan una relativa autonomía económica, política y tecnológica respecto del conocimiento científico provisto por el Estado o las empresas privadas. Desde esta perspectiva, la biodiversidad encierra en sí misma un proceso técnico y dinámico de selección y mejoramiento de especies cultivables (HernándezXolocotzi y Flores, 1971).

Ambos modelos coexisten en las superficies agrícolas mexicanas aunque sus funciones e intereses sean distintos. Con base en la idea de "modernización" propuesta en sus políticas de desarrollo agrícola, el Estado había destinado, en 2009, pagos al 56\% de los dos millones ochocientos mil campesinos maiceros, bajo el modelo agrícola-tradicional, estimados en el Censo agrícola y ganadero del INEGI del 2007 (Eakin et al., 2013). Las instituciones de desarrollo agrícola ${ }^{3}$ han orientado sus lineamientos científicos al mejoramiento de variedades vegetales con vocación altamente productiva mediante investigación en fitomejoramiento, biología molecular e intensificación productiva regional (Arellano y Ortega, 2005). Esto excluye a los campesinos indígenas, cuyas prácticas y lógicas están dentro de la agricultura tradicional.

Consideramos que la asistencia social por parte del aparato estatal se concentra en tres ideas: 1) la seguridad alimentaria —o combate al hambre-, basada en fortalecer los esquemas de aprovisionamiento y comercialización de productos de la agricultura; 2) la modernización del campo, basada en la idea de renovación productiva e inserción al mercado a través de la promoción de cambios culturales y de nuevas vocaciones productivas, y 3) el cambio climático, entendido como el apoyo a proyectos estratégicos de generación de energía renovable, mercados de carbono y servicios ecosistémicos en regiones que, por estar en situaciones de riesgo, son definidas como prioritarias. 
El objetivo de esta comunicación es categorizar los valores e ideologías que manifiestan los actores técnicos, productivos, políticos, asociativos, académicos y económicos relacionados con la soberanía alimentaria ${ }^{4}$ en Los Altos de Chiapas. Para ello, se puso en el centro de la discusión el programa de desarrollo agrícola promovido por instancias gubernamentales denominado Programa Maíz Solidario (en adelante PMS). Se propone un marco de referencia teórico proveniente de la sociología de la traducción de Callon y Latour (Callon, 1986; Latour, 2005; Callon, 2006a; Callon, 2006b; Callon et al., 1995; Callon et al., 2006), a partir de los conceptos de paradigma tecnológico, redes sociotécnicas, polos y actores. Las diversas posiciones de los actores permiten identificar los intereses y retos para la conservación campesina de maíces criollos en la región de Los Altos y entender el contexto actual en el que están inmersos los modelos de conservación aquí descritos. Los resultados de este artículo se basan en un panel sobre "Conservación de maíces criollos" realizado en octubre de 2011, en el que participaron investigadores especializados en maíz, en Chiapas, de instituciones como el Centro de Investigaciones y Estudios Superiores en Antropología Social (CIESAS) y el Colegio de la Frontera Sur (ECOSUR), representantes de organizaciones que trabajan en la producción de maíz, productores de diferentes municipios de Los Altos ${ }^{5}$-Tenejapa, Amatenango del Valle y Teopisca - y actores del sector público, como la Secretaría de Medio Ambiente y Recursos Naturales-Comisión Nacional de Áreas Naturales Protegidas (SEMARNAT-CONANP), el Instituto Nacional de Investigaciones Forestales, Agrícolas y Pecuarias (INIFAP), la Secretaría del Campo del Estado de Chiapas (SECAM) y la Secretaría de Pueblos y Culturas Indígenas - antes Secretaría de Pueblos Indios-. Asimismo, la información relativa al contexto histórico del establecimiento del PMS se recabó mediante una docena de entrevistas con informantes de organizaciones sociales, instituciones académicas e instituciones públicas, de quienes se conserva la confidencialidad de su identidad.

\section{El contexto del PMS y los actores implicados}

La agricultura tradicional de Los Altos de Chiapas es practicada mayoritariamente por indígenas y se ubica —dados los desplazamientos y dinámicas poblacionales históricas ligadas a la competencia por la tierra- en geografías accidentadas, montañosas y de difícil acceso, por lo que desde la perspectiva de los funcionarios públicos es ante todo una agricultura deficitaria, ineficiente, de baja producción y por lo general difícil de atender gubernamentalmente. Sin embargo, la biodiversidad cultivada es propia de la agricultura tradicional ya que remite a un sistema de asociaciones y relaciones vegetales de sistemas productivos como la milpa ${ }^{6}$ el huerto familiar y las tierras de acceso colectivo -bosques, selvas o acahuales-, que constituyen espacios altamente generadores de biodiversidad (Mariaca et al., 2010).

Se ha comprobado que la biodiversidad de maíces nativos está asociada a la diversidad cultural (Bentz, 1997; Boege, 2010). En el año 2000, en Chiapas, se sembraba casi un millón de hectáreas de maíz, con una producción cercana a los dos millones de toneladas, por lo que este estado se constituyó en el tercer productor en el ámbito nacional. En esta actividad participaban cerca de medio millón de hogares (Perales y Hernández-Casillas, 2005). En Chiapas están registradas más de veinte clases de maíz, de las cuales once son las más comunes (Perales y Hernández-Casillas, 2005). En la región de Los Altos hay presencia de seis variedades, de las cuales tres —olotón, olotillo y comiteco - son las más frecuentes. Estas razas de maíz son cultivadas principalmente por pequeños productores de poblaciones indígenas pertenecientes a dos grupos étnicos mayoritarios, el tsotsil y el tseltal.

En los últimos cincuenta años, la región de Los Altos $^{7}$ ha estado influida por un proceso de control cultural (Köhler, 1975), que se ha agudizado a partir de la incidencia del paradigma neoliberal. A partir del año 2000, en Los Altos, aumentaron los procesos de migración campesina, lo que coincidió con la introducción de una cantidad creciente de programas de corte agrícola, ${ }^{8}$ con nuevos componentes técnicos y procesos de organización de los grupos de productores 
rurales. Con dichos componentes técnicos se intentaba dar una respuesta productiva a las zonas templadas, donde no hay acceso a la producción de café, cuyos habitantes vienen de un proceso histórico de pobreza y marginación económica y alimentaria ${ }^{9}$ dada la erosión del suelo, la baja productividad y la reducción de las áreas cultivadas con milpa. Resultado de este tipo de intervenciones ha sido la aparición de lo que puede identificarse como dos grandes tipos de campesinos; uno de ellos, al que llamamos "neo-milpero", listo para diversificar sus sistemas productivos mediante la introducción de invernaderos con cultivos orgánicos, generalmente de mayor rentabilidad —en el mejor de los casos-; y un segundo tipo, al que llamamos "micro milpero", listo para emigrar, rentar o vender sus tierras, quien abandona la agricultura en aras de su integración económica a las ciudades. Denominamos a los dos tipos "milperos" porque siguen utilizando como base alimentaria los cultivos que conforman el concepto original de la milpa: maíz, frijol y calabaza.

En el año 2000, el gobierno de Chiapas creó el Consejo Distrital de Desarrollo Rural Sustentable de Los Altos, en el que convergían tres posiciones políticas cuyos diagnósticos sobre la realidad agrícola respondían, en términos generales, a tres modelos ligados a sus propios intereses: uno asistencialista, con el que se buscaba el respeto a las cuotas, formas y actores "institucionales" en la asignación de recursos; otro empresarial, que se enfocaba en los actores eficientes en la intensificación productiva; y un tercero, que se encaminaba hacia los pequeños campesinos, y con el cual se proponía un esquema de intensificación moderado que se basaba en el maíz nativo como eje productivo. ${ }^{10}$ Entre 2006 y $2007,{ }^{11}$ en un contexto de transición y cambio de poderes para el períodogubernamental estatal, entre 2006 y 2012, el recién electo gobierno lanzó el PMS, cuyo objetivo principal era "fortalecer y alcanzar la seguridad alimentaria en la entidad". ${ }^{12}$ Las posturas sobre las soluciones para fortalecer la seguridad alimentaria dentro del Consejo eran divergentes en tanto que apuntaban a los tres modelos de agricultura descritos anteriormente, por lo que, en opinión de uno de los actores académicos que participaron en el Consejo, con el PMS se intentaba conciliar los modelos utilizando el maíz como eje de desarrollo y buscando aumentar su productividad. El programa consistía originalmente en la dotación de 170000 paquetes tecnológicos - con un precio de mil pesos cada uno-, que supuestamente respondían a una demanda de las comunidades indígenas y campesinas y a los lineamientos desarrollados en el Consejo como una solución al problema de la baja fertilidad de los suelos, la incidencia de malezas ${ }^{13}$ y la baja calidad nutricional de los maíces sembrados por la población de Los Altos. Los insumos para estos objetivos consistían en un paquete de cincuenta kilos de fertilizante químico (NPK), veinte litros de plaguicida, un costal de cincuenta kilos de herbicida y veinte kilos de semilla híbrida de maíz, denominada Quality Protein Maize (QPM). ${ }^{14}$ La SECAM era la responsable de la dotación de los paquetes; sin embargo, la entrega directa no corría a cargo de la SECAM, sino de las comercializadoras y distribuidoras oficiales designadas por el gobierno estatal.

En la región de Los Altos, la dotación estaba destinada a los municipios de Zinacantán, San Andrés Larráinzar, Tenejapa, Teopisca, Mitontic, Chenalhó y San Juan Cancuc. El padrón de beneficiarios lo realizó la SECAM con el apoyo de la Secretaría de Pueblos Indios (SEPI), ${ }^{15}$ en coordinación con las agencias, asociaciones y organizaciones no gubernamentales que representaban a los productores organizados de dichos municipios. La dotación de agroquímicos y de semilla QPM generó una gran oposición en las organizaciones que supuestamente representaban al grupo de campesinos de Los Altos dentro del Consejo, pero también en los ciudadanos de San Cristóbal de Las Casas, quienes en esos mismos años se habían constituido en una iniciativa de consumidores conocida como Comida Sana y Cercana, cuyo objetivo era encontrar productores orgánicos para abastecer a sus familias de alimentos sanos y cultivados con aguas limpias. ${ }^{16}$

El gobierno estatal a través de la SECAM realizó una serie de negociaciones con las organizaciones y asociaciones opositoras al PMS, constituidas en Iniciativa Popular de Maíz Criollo, y, posteriormente, con la Red de Defensa del Maíz Criollo de Chiapas (Gómez, 2012). Las organizaciones proponían la anulación del 
reparto de paquetes químicos - por atentar contra el proyecto alimentario y productivo, el cual venían defendiendo desde hacía varios años- y la creación de un sistema micro-regional de fitomejoramiento participativo y de comercialización de semillas orgánicas a través de la participación de cooperativas agrícolas, el mejoramiento y la restauración de suelos mediante técnicas agroecológicas, la introducción de frutales y el uso de insumos orgánicos como micorrizas y humus. ${ }^{17}$ Algunas de las organizaciones y asociaciones civiles que se enfocan hacia el desarrollo rural - situadas en su mayoría en San Cristóbal de Las Casas- propusieron que se retomaran los componentes orgánicos de conservación de semillas al estilo del Programa Tecnológico de Cultivo de Maíz Orgánico que la SAGARPA había implementado en $2003,{ }^{18}$ junto con cursos de fitomejoramiento participativo de maíces nativos y la transformación de productores en "técnicos comunitarios". Asimismo, varios de los actores que representaban a las organizaciones sociales de la Red de Defensa del Maíz Criollo de Chiapas (RMCC) presentaron una propuesta para la entrega de subsidios directos a los productores de la milpa, por ser éstos los custodios de un sistema tradicional de producción de maíces nativos.

Se logró un acuerdo entre gobierno y organizaciones en el que se favorecía a ambos modelos de agricultura; es decir, a las organizaciones que aceptaban la propuesta del maíz como eje de desarrollo, con una producción apoyada con agroquímicos, y a aquellos que optaron por una producción de maíz moderada en agroquímicos, o de preferencia de tipo orgánico, dirigida a pequeños productores. Por un lado, se respetaban los compromisos que formal o informalmente tenía el gobierno estatal tanto con los productores de maíz altamente dependiente del uso de agroquímicos, como con las comercializadoras y distribuidoras de estos insumos. Por otro lado, se acordaba que a los productores tradicionales, representados por la RMCC, se les entregarían abonos orgánicos en lugar de fertilizantes químicos y, en lugar de semillas QPM, se les haría el pago correspondiente por ser productores de semilla de maíz nativo.
Los acuerdos entre las organizaciones y el gobierno sobre las proveedoras de paquetes orgánicos y sobre el pago a productores fueron informales o de palabra, sin que existieran minutas de las reuniones, ${ }^{19}$ por lo que, en términos prácticos, surgió una serie de problemas cuando las organizaciones iniciaron las modificaciones propuestas: 1) la operatividad burocrática-administrativa de las instituciones del estado no permitía realizar compras de semillas sin una factura; 2) los campesinos no tenían posibilidad de darse de alta a corto plazo ante Hacienda Pública, ni de facturar su trabajo como productores "orgánicos" o como "custodios de la biodiversidad", a fin de recibir el pago estipulado; 3) los marcos normativos establecidos en la Ley de Variedades Vegetales no permitían la venta de semillas sin la certificación del Servicio Nacional de Inspección y Certificación de Semillas (SNICS); 4) los campesinos de Los Altos no podían certificar sus maíces nativos como variedades o razas, ya que los protocolos del SNICS son bastante exigentes a fin de garantizar la estabilidad y homogeneidad de las semillas, y 5) las limitaciones propias de la agricultura campesina de Los Altos para generar excedentes de semillas y asegurar su comercialización.

A decir de algunos actores de las organizaciones sociales, existió un proceso de exclusión que caracterizó la toma de acuerdos sobre la operación del programa y sobre quiénes serían los proveedores oficiales de los insumos, particularmente de las semillas nativas. Las decisiones fueron tomadas por dos o tres interlocutores de la RMCC, quienes se reunieron con los interlocutores institucionales sin acuerdo de la mayoría, asumidos como representantes del resto de las organizaciones y sin tener, en opinión de las organizaciones excluidas de la negociación, una representatividad efectiva en cuanto a sus puntos de vista e intereses.

En septiembre de 2008, al concluir la negociación entre la RMCC y la SECAM, se entregaron doscientos mil paquetes tecnológicos, de los cuales cincuenta mil fueron con abonos orgánicos y sólo cuatro mil incluían el pago a productores de la RMCC por uso y producción de semillas nativas. El resto 
de los paquetes siguió incluyendo componentes agroquímicos y semillas híbridas. Dentro de la operación del programa para esos cuatro mil paquetes tecnológicos, se incluyó la entrega de un "cupón de consumo" para cada productor inscrito en el padrón de la SECAM por un valor de mil pesos. Los cupones se intercambiaban por productos orgánicos o químicos ofrecidos por las comercializadoras oficiales. Las comercializadoras recibían los cupones y, a cambio, los campesinos admitían insumos orgánicos o químicos - según la elección de cada productor - por el monto de seiscientos pesos. Adicionalmente, las comercializadoras entregaron trescientos pesos en efectivo a cada productor como "pago por su trabajo en la producción de semillas criollas”.

Las comercializadoras debían presentar los cupones ante la SECAM y, en contrapartida, recibían novecientos pesos por cada uno. Esta operación se respaldaba - en términos fiscales - con la facturación de mil pesos por cupón. Sin embargo, en esta transacción existe una aritmética inconsistente ya que no se vislumbra cómo las comercializadoras podían facturar por mil pesos al gobierno, en tanto que los productores sólo recibían novecientos pesos - seiscientos pesos en insumos y trescientos de pago en efectivo-. A decir de algunos de los actores que participaban en las organizaciones sociales, estos cien pesos que parecían "perdidos" fueron utilizados como costos de implementación y capacitación técnica por las organizaciones que participaron en la negociación. Otros dicen que funcionaron como una comisión para los funcionarios estatales que diseñaron y agilizaron el esquema de operación.

Aún en diciembre de 2013, el PMS seguía distribuyendo paquetes tecnológicos, bajo el nombre de Programa de Maíz Sustentable, pero los pagos a los agricultores ya no se llevaban a cabo. ${ }^{20}$ Las apreciaciones sobre la legitimidad de la representación en la negociación, la forma en que se alcanzaron los acuerdos entre el gobierno y las organizaciones, y las sospechas de corrupción ligadas al establecimiento de los paquetes, hicieron que las organizaciones que participaban en la RMCC se dispersaran.

\section{Aproximación al PMS desde la noción de redes sociotécnicas}

La sociología de la traducción ${ }^{21}$ ofrece un marco de comprensión sobre las relaciones de diferentes actores de la innovación dentro de una realidad, donde el motor de la innovación se basa en intereses económicos y en su aceptación dentro de un mercado (Callon, 2006a; Callon, 2006b; Callon y Latour, 1985; Latour, 2005). Los elementos que analiza la sociología de la traducción para caracterizar los procesos de innovación son los paradigmas tecnológicos, la trayectoria tecnológica y la cadena de innovación, entendida esta última como la red sociotécnica.

El paradigma tecnológico está descrito como "la selección" de problemas tecnológicos. La selección es entendida como la construcción de un proceso social en el que intervienen distintas medidas para plantear una solución (Dosi, 1998 en Montaigne, 1997: 10). La solución está marcada por cuestiones que tienen que ver con cuál, qué, dónde, cómo y por qué se define un problema. Por otro lado, la decisión sobre lo que se identifica como problema también está en función de quiénes toman las decisiones, así como de los principios que rigen la organización para el trabajo. Es relevante el peso que tienen los cuerpos de conocimiento aplicados para resolver el problema, así como los materiales y desarrollos tecnológicos pertinentes para generar soluciones. El paradigma tecnológico se construye a partir de los programas de investigación científica. Podemos decir que el hardware del paradigma es el de "las instituciones, disciplinas profesionales, empresas públicas o privadas y universidades" (Dosi, 1988), mientras que el software son "los programas de investigación-formación, líneas estratégicas, normas y reglamentos legales que sostienen el desarrollo tecnológico" (Dosi, 1988). Como se puede apreciar, el paradigma tecnológico tiene toda una plataforma político-económica detrás que se manifiesta a través de un hardware y un software institucionales para ofrecer soluciones a problemas consensuados o ya decididos. El paradigma es constantemente evaluado por un grupo de actores que participan dentro de la estructura de 
beneficios político-económicos, quienes lo replicarán en tanto sus intereses no se vean afectados.

Parte de la dinámica descrita en los procesos de innovación técnico-científica reside en la habilidad para destrabar los obstáculos denominados "controversias", generados entre los actores que participan en la aplicación de un paradigma. Las controversias se resuelven a través de procesos de negociación (Callon, 2006a) desde el momento en que se identifican los intereses de los actores y existe la voluntad de llegar a una convención donde todos, o una mayoría, lleguen a un acuerdo favorable.

Las controversias pueden comprenderse a partir de la interpretación que hace una serie de actores ligados a una implementación sociotécnica en particular. La noción de red sociotécnica explica el conjunto de relaciones heterogéneas dentro y entre los polos de transferencia y desarrollo de la innovación, representados por el hardware. En el caso que aquí se plantea hemos reconstruido las controversias que incidieron en la trayectoria del paradigma tecnológico, representado por la diversidad de actores que participan en una implementación técnica y social dentro de una red sociotécnica (Callon et al., 1995).

La red sociotécnica tiene diferentes "polos" que se expresan en los intereses, discursos y objetivos de los actores que los representan. Sobra mencionar que los actores pueden no compartir los mismos discursos o valores para ser catalogados como homogéneos, legítimos, o de buena o mala moralidad. Las nociones de polos y red sociotécnica nos sirven para describir las controversias implícitas en el establecimiento de un programa gubernamental de orientación agrícola cuyo objetivo era la repartición de paquetes tecnológicos conformados por semillas de maíz mejorado en comunidades campesinas de Chiapas. ${ }^{22}$ Las controversias identificadas se expresaron en el panel de diálogo "Conservación de Maíces Criollos", realizado en octubre de $2011 .{ }^{23}$ Dicho foro contó con la presencia de varios de los actores implicados en la negociación y contestación del programa. Aunado a ello, se realizaron diversas entrevistas, posteriormente, con otros actores implicados tanto de la academia como de las organizaciones sociales y ex funcionarios de la SECAM.

\section{Polos y tipologías de los actores en el PMS}

En este apartado se presentan y describen los actores gubernamentales, científicos y productivos que participaron en la implementación técnica y social de la innovación. Los polos de transferencia y desarrollo de la innovación, descritos por Callon (1995), son los polos científico, técnico, institucional, de mercado y productivo. Como parte de nuestro análisis decidimos crear una serie de tipologías para definir los polos con mayor detalle. Los nombres y asignaciones de cada subtipo descritos en este apartado son una propuesta de los autores de este artículo.

\section{Polo científico}

Se caracteriza por la producción de conocimientos que realizan los laboratorios o centros de investigación, ya sean industriales, académicos o empresariales. Los actores son científicos, investigadores o académicos. En el caso del PMS, el polo no es homogéneo, por lo que se propuso una tipología de tres subgrupos:

El primer subgrupo lo identificamos como los "cuantitativistas" y está conformado por investigadores cercanos a las ciencias exactas - agronomía y biología-, quienes pueden trabajar en coordinación con instituciones nacionales e internacionales para evaluar indicadores muy precisos sobre programas o fenómenos de interés biológico relacionados con la adaptación y el cambio climático. Asumen una posición neutral sobre la innovación y la tecnología. Tienen una formación académica doctoral y sus espacios de divulgación científica se dan en el ámbito internacional.

Un segundo subgrupo es el denominado "instrumentalistas". Está conformadopor desarrolladores con formación profesional cercanos a las instituciones públicas y a la estructura gubernamental. Tienen amplias redes sociales al interior del gobierno y son empleados pensionados y personal activo que ocupó puestos de dirección, vinculados a la toma de decisiones. 
Sus posiciones son cercanas al desarrollo y a la aplicación de agroquímicos, variedades mejoradas o introducción de transgénicos. Son cercanos a las instituciones de mejoramiento genético y experimentación agronómica, y en algunos casos pueden acceder a utilizar la infraestructura, así como los recursos humanos y técnicos, para proyectos económicos personales. ${ }^{24}$ Tienen interlocución fluida con instituciones federales y privadas. Están conformados en cuerpos colectivos amplios, como asociaciones y colegios estatales de agrónomos y genetistas.

El último subgrupo lo identificamos como los "bioculturalistas". Son los investigadores ligados a redes de agroecología nacionales e internacionales, que tienen un enfoque más cualitativo y cercano a la agricultura tradicional. Su formación puede ser en agronomía o biología, pero son próximos a disciplinas sociales como la antropología y la sociología. Están interesados en los fenómenos sociales de tipo local ligados a la biodiversidad, los saberes tradicionales y su transmisión generacional.

\section{Polo técnico}

Se centra en los dispositivos capaces de ofrecer servicios específicos que implican la realización de un programa de acción. Los actores de este polo son ingenieros, técnicos o, en el caso que nos compete, algunas organizaciones o asociaciones civiles que cumplen funciones de capacitación y extensión. ${ }^{25}$ Nos referimos como técnicos a aquellos que cumplen alguna función relacionada con ofrecer un conocimiento nuevo al beneficiario y que incide, de alguna manera, en la resolución de un problema específico. Dos subgrupos fueron identificados, de los que proponemos la siguiente tipología:

El primero, que denominamos "tecnocrático" por estar sus integrantes subordinados salarialmente a la política estatal, está conformado por quienes, además de considerar la milpa como un espacio de conservación, tienen un contacto cercano con actores técnico-administrativos gubernamentales y son conocedores de las políticas públicas, los programas gubernamentales y los lineamientos operativoadministrativos propios de la estructura estatal. Está conformado por organizaciones ${ }^{26} \mathrm{y}$ asociaciones ligadas a la agricultura tradicional, los derechos económicos, sociales, culturales y ambientales; $y$ son actores políticos cuyos ingresos dependen fuertemente de la gestión de programas gubernamentales.

El otro subgrupo, denominado "activistas", remite a las organizaciones que proporcionan asesoría técnica ligada a la agricultura ecológica pero que son ajenas al juego de intereses técnico-económicos o de arreglos interinstitucionales. Se trata de organizaciones más consolidadas que las "tecnocráticas", en tanto que tienen una planta de recursos humanos con contratos temporales - no dependientes del voluntariado- y sus empleados son originarios de las regiones donde intervienen. Son organizaciones que tienen un discurso agroecológico, capacidad para diseñar proyectos de capacitación y autogestión, y recursos y apoyos de agencias de cooperación internacionales. A diferencia de los tecnocráticos, los activistas llevan a cabo procesos de intervención técnica con ciclos más largos y un seguimiento más continuo. No están exentos de participar en programas gubernamentales, pero prefieren estar ligados a programas y fondos privados o del exterior.

\section{Polo mercado}

Este grupo hace referencia a los actores que participan en los intercambios económicos y en la circulación de información. Los actores en este polo son los usuarios de las tecnologías, las empresas proveedoras, las redes de distribución y de comercialización, pero también las asociaciones de consumidores. Este grupo incluye las empresas de comercialización y distribución de insumos que normalmente participan en programas de gobierno, las subsidiarias o comercializadoras de agroquímicos - frecuentemente de trasnacionales-, y las empresas de tipo local que venden insumos considerados alternativos o biológicos, pero igualmente provenientes de un sistema de producción bajo el mismo paradigma comercial. También se refiere a los 
consumidores de los productos agrícolas, quienes pueden estar representados por asociaciones de consumidores interesadas en fomentar la producción y el consumo de productos “orgánicos”. Nos referimos a la iniciativa ciudadana Comida Sana y Cercana, integrada por personas ligadas a la academia o centros de investigación, universidades y programas de posgrado de San Cristóbal de Las Casas. ${ }^{27}$ Este grupo fue invitado a participar en el Panel de Conservación de Maíces $\mathrm{y}$, aunque algunos de sus representantes asistieron y vertieron sus opiniones, prefirieron no actuar como asociación de consumidores, sino como consumidores en general.

\section{Polo institucional-estatal}

Se caracteriza por la orientación política de los programas de tipo productivo. Los actores de este polo son directivos o funcionarios de gobierno que guardan una línea congruente con la política del Estado. Son actores intermediarios entre la ciencia y la técnica. Este polo estuvo representado por actores que nosotros denominamos "vocacionales", en contrapartida con los funcionarios, directivos o agentes institucionales que fueron seleccionados por sus capacidades como dirigentes, académicos o mediadores de movimientos sociales.

\section{Polo productivo}

Se refiere a las organizaciones productoras o a los actores organizados, ya sean privados o colectivos, que funcionan individualmente o en forma cooperativa. Este polo está representado por al menos tres subgrupos de actores: ${ }^{28}$ uno "caciquil", que pretende que el gobierno estatal les entregue recursos económicos directamente; otro "empresarial", que busca que los recursos se destinen a un número pequeño de productores que ha mostrado tener eficiencia productiva; $y$ un tercero, "milpero", que defiende la producción de maíz a través de la intensificación productiva sin la introducción de agroquímicos en la milpa tradicional. Solamente este tercer grupo tuvo representación en el foro.

\section{Siete controversias capitales}

Las controversias presentadas surgieron a partir de la discusión de diferentes aspectos del proceso para el establecimiento del PMS. Los discursos de los actores participantes en el panel fueron transcritos y analizados con apoyo del programa Atlas-ti. En él se hizo la codificación de las categorías que salían a relucir en el discurso con categorías previamente definidas, tomando en cuenta los principales puntos de desacuerdo. El programa permitió crear una matriz de conexiones entre los argumentos de los actores para conocer dónde había mayor densidad e intercambio de puntos de vista. Los autores identificamos y propusimos los argumentos que consideramos clave. ${ }^{29}$ Es decir, aquellos argumentos que consideramos que despertaron una respuesta contradictoria, crítica o polémica entre actores. Decidimos presentar las controversias en un orden que consideramos lógico y que facilita la lectura, por lo que, desde nuestro punto de vista, no existe una controversia más importante que otra. A continuación presentamos el análisis agrupado en las siete controversias que consideramos más importantes o capitales para entender la postura al interior de la red sociotécnica que implementó el PMS.

\section{Controversia l: las denominaciones del maíz criollo}

Una de las discusiones se refiere a las diferentes concepciones de maíz criollo. Para los bioculturalistas, el término "maíz criollo" alude a algo foráneo y contradictorio ya que México es un país donde se produce maíz y todo el maíz es local. Lo "local" es lo indígena. La acepción más usada por los bioculturalistas es "maíz nativo", lo que conlleva representaciones culturales y geográficas. Para los cuantitativistas, el "maíz nativo" también es el término más común; sin embargo, consideran que no es un concepto preciso por posibles procesos de hibridación con materiales de otros lugares o de tipo comercial. Señalan que son maíces híbridos o hibridizados cuando provienen de la práctica premeditada de experimentación por parte del agricultor. 
Otro tipo de maíz es el "acriollado", aquel que el campesino cruza, por lo que, genéticamente hablando, a decir de los instrumentalistas, su origen "nativo" es difícil de identificar; por ello, se refieren a estos maíces como "no criollos". Maíces "comerciales" son aquellos cuyo proceso de desarrollo genético ha sido realizado por una estructura científica - pública o privada- y están ligados a una estructura comercial de distribución. Para este grupo es más preciso hablar de maíces en términos de razas, para clasificar las características fenotípicas, aunque a decir de los cuantitativistas esta definición resulte un tanto artificial o subjetiva. Otra noción propuesta por el polo técnico-tecnocrático se refiere a los maíces "mestizos" como aquellos que han sido polinizados con nativos o con otros cuyas formas de manejo son diferentes a la campesina. También proponen la noción de maíces "tradicionales" u "originarios" cuando no han sido polinizados por un maíz proveniente de un proceso científico y "popular", según el sector que maneja generalmente los recursos genéticos.

\section{Controversia 2: mejoramiento isegún quién?}

La diversidad de términos refleja un aspecto relativo a cómo los actores perciben la "otredad del maíz" y que, desde el lado de la ciencia, se insiste en los términos de "pureza" y "mejoramiento", términos también ambiguos que incluyen, unilateralmente, una serie de principios para definir quiénes son los capacitados para avalar qué es lo "puro" y qué significa “mejorar". Para el campesino, mejorar implica un proceso de experimentación, "hacer algo útil" para los fines que ellos deseen. La utilidad la definen en cuanto a la resistencia a las plagas o al viento, el peso del grano, el sabor y la energía que les aporta el maíz.

Por su parte, los científicos instrumentalistas se autoasumen como quienes avalan los procesos de mejoramiento. Para ellos, los campesinos "mejoran la semilla" a través de la polinización entre maíces comerciales - provenientes del quehacer científicocon los maíces campesinos.

Los instrumentalistas consideran el mejoramiento como un proceso complejo que se realiza en un laboratorioo en un campo experimental por ungenetista. Los campesinos son solamente los reproductores de la variabilidad de maíces, lograda a través de los procesos científicos de mejoramiento. Estas opiniones reflejan que es la autoridad del científico, y no la del campesino, la que califica qué se entiende por mejoramiento, raza, pureza, contaminación, híbrido o nativo.

Para los cuantitativistas, el proceso de mejoramiento está relacionado con la experimentación y con los procesos de hibridación o acriollización que realiza el campesino. El término mejoramiento implica el conocimiento basado en el método científico, mientras que el saber indígena y campesino tiene sus limitaciones y es incuantificable. Este grupo es el primero en señalar el tema de las semillas transgénicas como un proceso de cruzamiento genético "no buscado" por los campesinos y, por lo tanto, como un riesgo de erosión genética para los maíces nativos.

Los tecnocráticos y activistas coinciden en señalar que el mejoramiento es un proceso de conocimiento campesino y que existen procesos de experimentación que efectivamente son premeditados, mientras que otros no lo son. Para ellos, el campesino no utiliza o no comprende en la misma dimensión conceptual la pureza o la contaminación - ambos conceptos, abstracciones de la biología-. Coinciden en señalar que existe peligro de contaminación transgénica en los procesos de mejoramiento campesino o en los procesos de hibridación no buscados.

Finalmente, los bioculturalistas coinciden en que el mejoramiento, concepto propuesto por los cuantitativistas, es una cuestión que realizan los campesinos y son ellos quienes deben ser considerados como los mejoradores del maíz, aunque su método no sea el científico. Según su posición, el mejoramiento es un proceso dinámico, propio de la racionalidad campesina tradicional.

\section{Controversia 3: ide qué conservación estamos hablando?}

Los argumentos anteriormente descritos muestran cómo cada actor tiene una comprensión diferente sobre el mejoramiento y, por lo tanto, sobre lo que significa 
conservar. Para los bioculturales, la conservación es la reproducción de las especies biológicas nativas, al mismo tiempo que las relaciones socioculturales entre quienes las producen. Los cuantitativistas, de forma similar, consideran que la conservación es el proceso de mediación entre la experimentación con variedades nativas y la búsqueda de mejoramiento que los campesinos pretenden. La conservación significa mantener las condiciones para que persista la experimentación campesina, por lo que el acriollamiento es una forma de conservación de materiales genéticos. Por su parte, los instrumentalistas consideran que son las instituciones públicas y los programas de gobierno los que permiten la conservación y que ésta debe mantener la pureza de las razas para que continúe habiendo homogeneidad en las especies y un acervo con características diversas.

En el polo técnico existen algunas posiciones similares; es así como los tecnocráticos y los activistas coinciden en que la conservación implica evitar la contaminación transgénica. En ese sentido, la pureza no es importante siempre y cuando los genes pertenezcan a una misma especie y provengan de un método campesino de siembra, cosecha y reproducción tradicional de la semilla, ciclo tras ciclo agrícola. La práctica de conservar el maíz conlleva mantener físicamente los espacios de reproducción - milpa y acahual- Para el polo estatal, la conservación es un aspecto de apropiación y control de los factores culturales. El conservacionismo parte de la idea de mantener el maíz en un banco de germoplasma, casi de la misma forma en que el gobierno quisiera mantener a los pueblos indígenas en una vitrina, a fin de mantenerlo "puro". Finalmente, para los productores maiceros, conservar es fortalecer la semilla que les dieron sus padres.

\section{Controversia 4: los agentes de la innovación}

Existen diferentes perspectivas respecto a lo que es la innovación y cómo se expresa según el tipo de polo y de actor. En el polo científico, los instrumentalistas piensan que la innovación es el proceso de fitomejoramiento. Desde su perspectiva, los campesinos deberían ajustar sus prácticas a los procesos de innovación tecnológica que ellos promueven, ya que las técnicas campesinas de producción no permiten que una misma parcela tenga la suficiente estabilidad genética de maíces a causa de la cercanía física y el cruzamiento no controlado. Los instrumentalistas consideran que la innovación es un proceso que ellos realizan y trasmiten. Innovar es intervenir genéticamente para incrementar los componentes nutricionales de maíces adaptados al clima o a las especificaciones de la industria. Por su lado, los cuantitativistas consideran que los esfuerzos de mejoramiento genético están en manos de la experimentación campesina, ya que los centros públicos son ineficaces para producir alternativas que acepten y utilicen los campesinos, más aún si se les compara con la efectividad que tiene la distribución y aceptación de las semillas provenientes de la industria semillera privada. ${ }^{30}$

Por su parte, los bioculturalistas consideran que la variabilidad existente de maíces es producto de las condiciones y prácticas de los campesinos, basadas en técnicas de clasificación de suelos, desarrollo de instrumentos para la actividad agrícola, diversificación de cultivos y control de plagas. La innovación para ellos es un proceso de fitomejoramiento participativo. Desde su perspectiva, las innovaciones también pueden ser de tipo político, como un posicionamiento ante la comprensión de fenómenos sociales complejos, como los transgénicos o la biopiratería.

Los campesinos se asumen como agentes de innovación genética en cuanto a la experimentación con maíces para la búsqueda de nuevos colores o el peso del grano. Proponen otras innovaciones técnicas de tipo agroecológico, como la conservación postcosecha o la preparación de la tierra sin RTQ — roza-tumbaquema-.

Los técnicos consideran que las innovaciones son metodológicas en cuanto a la organización, capacitación y difusión de prácticas agroecológicas. No existe mucha claridad sobre hasta dónde el técnico juega un papel en la innovación campesina, como en el caso de la experimentación genética.

Para los actores estatales vocacionales, la innovación se trata de un proceso social de adaptación a la 
matriz cultural de los pueblos, como por ejemplo la migración como estrategia de subsistencia, la búsqueda de mercados o rutas comerciales alternativas, o la creación de nuevos productos o formas diferentes de complementar su alimentación. Desde la perspectiva de estos actores, la innovación implica, igualmente, un proceso organizacional en tanto que reorganiza las formas sociales de producción en las comunidades.

\section{Controversia 5: iempoderamiento o dependencia?}

Cuando se habló en el panel del papel de los productores en la innovación, los actores iniciaron una discusión sobre si los campesinos, al estar fuera del esquema de subsidios y programas del Estado, estarían o no empoderándose.

Para los actores vocacionales, el tema de los subsidios es la "perversión" de una idea originalmente loable, donde el Estado apoya a los campesinos y, al mismo tiempo, un ejemplo del mal funcionamiento de las instituciones. Para los tecnocráticos, los subsidios representan un esquema que no beneficia a los pequeños productores sino que favorece a las multinacionales. Se refieren a los subsidios como "paliativos", "farsa", "engaño" o "condicionamiento"; los asocian al objetivo del gobierno de hacer eficientes a los campesinos mediante la reconversión productiva o de dejarlos simplemente desaparecer. Para los técnicos activistas, la reconversión productiva y los paquetes tecnológicos son una estrategia del gobierno para generar dependencia de los fertilizantes químicos, deudas impagables y, por lo tanto, migración forzada. Para los cuantitativistas, el papel del gobierno en el tratamiento del tema se refleja en la desaparición de los fondos económicos para la investigación sobre el maíz nativo por parte del Consejo Nacional de Ciencia y Tecnología. Desde su perspectiva, el Estado tiene una visión de menosprecio hacia el maíz como opción o eje del desarrollo. Igualmente, consideran que los subsidios tienen una lógica diferencial que funciona de forma poco equitativa dadas las limitaciones y condicionantes administrativas y burocráticas que impone el Estado.
Para los bioculturalistas, el abandono de la agricultura tradicional por parte del Estado propicia una cierta autonomía, dado que el campesino trabaja con sus propios recursos, sin apoyo ni asistencia técnica o créditos, lo que le obliga a resistir mediante la acción colectiva local. A nivel global, se presenta un movimiento colectivo en torno a la racionalidad campesina como una representación de sustentabilidad y de resistencia al modelo económico.

Los productores "maiceros" piensan que los subsidios les son denegados y que existe desatención por parte de las instituciones. Según su discurso, hay un sentimiento de olvido gubernamental, por lo que sólo pueden salir de su situación "luchando", dado que el gobierno "los está robando".

Los actores coincidieron casi en bloque en la idea de que el campesinado se encuentra ante una economía global y de ventajas comparativas en la que es más barato importar maíz y donde los subsidios representan únicamente una asistencia social y no una estrategia de fortalecimiento productivo ante la apertura comercial. En conjunto se manifestaron en contra del paradigma tecnológico institucional al señalar que las necesidades y demandas reales de los campesinos no se han resuelto. Según los discursos, el gobierno está en contra del maíz nativo y en contra de la agricultura tradicional, y manifiesta una constante incomprensión de lo que es el sistema de milpa y del campesino en sí mismo, ya que éste no es un agente de interés para la economía neoliberal por no ser sujeto de crédito ni un actor capaz de contribuir al incremento del consumo y del mercado.

\section{Controversia 6: sustentabilidad y modelos de agricultura}

Para los bioculturalistas, la conservación del maíz es parte de una racionalidad diferente ligada a la sustentabilidad. El campesino siempre ha estado preocupado por conservar lo que tiene para heredarlo a las generaciones siguientes, no sólo su semilla, sino todo su sistema productivo. Por esta razón, consideran que el campesino va a seguir jugando su rol en la conservación de las semillas. De ello depende la conservación de la agrobiodiversidad. En ese sentido, los cuantitativistas 
consideran que los campesinos deben ser los agentes que dominen el proceso evolutivo y de selección de los maíces, dado que no existe la capacidad organizacional ni estructural del Estado para asignar tal rol a otro actor. Para los cuantitativistas, el gobierno tiene un paradigma equivocado de la conservación del maíz, al ignorar su relevancia cultural, alimentaria y de bienestar de las familias campesinas.

El polo tecnocrático considera que el campesino es un actor de resistencia no sólo cultural, sino política, que contesta al gobierno y que se organiza para solicitar los cambios en los componentes de las políticas públicas que lo desfavorecen. Los técnicos vocacionales consideran que dicha resistencia política no es evidente, sino más bien muy sutil y que se manifiesta en los aspectos culturales ligados a la continuidad de su actividad agrícola.

Para los actores del polo estatal, los campesinos tienen claro su propio modelo de agricultura porque, aun aceptando que la asistencia técnica para la pequeña agricultura es prácticamente nula, los campesinos saben aprovechar los pocos programas de gobierno y darles un sentido productivo sin alterar sus prácticas culturales - en este caso la continuidad de la milpaPor su parte, los campesinos tienen poco qué decir teóricamente sobre las concepciones de modelos agrícolas. Su respuesta es completamente práctica: seguir en su actividad o dejarla en contra de su voluntad si es que la agricultura no les da para vivir y alimentarse.

\section{Controversia 7: el valor del maíz}

En cuanto a la valoración del maíz, los actores tienen diferentes puntos de vista sobre el tipo de valor que se le asigna. Para instrumentalistas y cuantitativistas, el valor es genético ya que la adaptabilidad en la amplia variabilidad de maíces es irreemplazable. En caso de perder las variedades de maíz, no habría semillas comerciales listas para ser liberadas en las zonas agrícolas ubicadas en altitudes por arriba de los 1400 metros sobre el nivel del mar.

Para los instrumentalistas, la variabilidad genética de los maíces tiene un valor de utilidad para la industria productora de tortillas; para los actores bioculturales, cuantitativos y tecnocráticos, el maíz tiene un valor cultural que se representa en la vida cotidiana, los conocimientos, la cosmovisión y la identidad que construyen los campesinos, además de la gran aportación culinaria de las familias campesinas que utilizan el maíz en una infinidad de recetas. También, los actores identificados como tecnocráticos hablan de su valor económico en términos de la balanza comercial de importación y exportación. Para los actores cuantitativistas, el valor económico también se representa en el valor de la producción total del maíz existente en las milpas de autoconsumo - el cual no es contabilizado- o en los costos de los subsidios de los programas productivos. Existe también —según los bioculturalistas, los vocacionales y los tecnocráticos - un valor simbólico del maíz como emblema contestatario o de resistencia indígena, donde el sistema milpa es un símbolo de autonomía alimentaria y de vida. Para estos mismos actores, el maíz tiene un valor político como contestación a las intervenciones públicas y un valor alimentario-nutricional en cuanto a la variedad de productos alimentarios que pueden producirse, a los saberes culinarios implícitos y a la seguridad alimentaria local que ofrece. Finalmente, los actores bioculturalistas reconocen un valor ecológico de los sistemas de barbecho asociados a la milpa en cuanto a que éstos permiten la restitución de la fertilidad con sistemas de regeneración, como los acahuales, en donde se reproducen cientos de especies vegetales y de fauna silvestre.

\section{Conclusiones}

En este artículo se expuso una descripción del funcionamiento del PMS, un programa técnico llevado a cabo por el gobierno estatal de Chiapas para campesinos indígenas en la zona de Los Altos. Los referentes teóricos y metodológicos provenientes de la sociología de la traducción - como la red sociotécnica, los polos de innovación y el paradigma tecnológico institucionalnos permitieron organizar y analizar datos sobre la aplicación y las perspectivas de los diferentes actores 
que participaron de alguna forma en dicho programa, cuya operación muestra que, efectivamente, los dos "modelos" propuestos como referentes teóricos para entender la conservación de la biodiversidad, el biotecnológico y el agrícola-tradicional, permiten sistematizar las controversias centrales entre los interlocutores que participaron en la discusión.

Entre los polos de los modelos biotecnológico y de agrícola-tradicional existen suficientes variantes como para crear nuevos submodelos más precisos que describan las interrelaciones entre los polos científico, técnico, estatal y productivo para la conservación.

Al tipificar a los actores que intervienen en los polos mediante la noción de red sociotécnica, se observa que dicho referente teórico, proveniente de la sociología de la innovación y de la economía institucional, resulta útil para la descripción del proceso de flujo de información entre actores. Nos parece que es un referente al que se le puede sacar mayor utilidad cuando se aplica en regímenes tecnológicos más rígidos y jerárquicos, donde no exista tanta intervención de factores sociales poco controlables. Esta noción es muy útil en términos de registro y seguimiento de las decisiones, como en el caso de un laboratorio científico o una empresa de alta tecnología. Sin embargo, al aplicarse al caso de un programa agrícola, el concepto de red sociotécnica presenta algunas limitaciones ya que no permite precisar las formas de organización y de decisión en grupos sociales que no se ajustan a una lógica de mercado, como es el caso de los discursos culturales o de las construcciones propias de cada grupo étnico, ni aquellas consideraciones de tipo antropológico - reglas de intercambio de semillas entre linajesque están altamente presentes en el manejo de la agrobiodiversidad de las comunidades indígenas de Los Altos.

Otra limitación del concepto de red sociotécnica es su incomprensión sobre las cuestiones de estratificación social en una sociedad en particular. Idealmente, la red sociotécnica exige un diálogo donde las partes estén igualmente representadas y los actores sean lo más objetivos posibles. En la realidad de la cadena de la innovación aquí presentada, dentro del PMS, vemos un constante esfuerzo gubernamental asimétrico que utiliza la innovación como un medio de control cultural en las relaciones entre el gobierno y los indígenas campesinos. Es probable que, para el caso agrícola en donde desarrollamos tipologías de los agricultores, la noción de clase social pudiera ser útil para complementar la aceptación o el rechazo de una innovación y explicar por qué algunos actores fueron excluidos de la toma de decisiones en la modificación del programa. En ese sentido, la noción de clase social expone la asimetría de las relaciones sociales y nos introduce en las relaciones de poder asimétricas dentro de la red.

Nuestra tipificación de los actores guarda coherencia con la propuesta de red sociotécnica de Callon y Latour, ya que nos permitió agrupar en polos las posiciones de los actores, las cuales en un principio eran difíciles de comprender. A partir de esta tipificación se pudo comprobar que la intervención tecnológica dentro del programa estatal analizado no es neutral ni ajena a intereses, sino que responde a un paradigma tecnológico específico que está expresado en las relaciones entre actores y en las controversias aquí expuestas. Las imbricaciones entre ciencia, tecnología y lo social dentro del PMS no parecen responder a la problemática ni a los valores y objetivos de los campesinos indígenas tomados como beneficiarios, sino a intereses económicos de grupos ligados a la estructura gubernamental.

Las discusiones entre los actores dieron origen a una rica cantidad de representaciones sobre lo que para ellos significa el maíz criollo, el mejoramiento, la conservación y la valoración. Esto apunta a una discusión profunda sobre el tipo de conocimiento que se contrapone y sobre todo para discutir cuestiones como las siguientes: ihacia quién está dirigida la tecnología, tomando en cuenta que el conocimiento proviene de diversos polos y se dirige a diferentes actores?, ¿hasta dónde son válidos y útiles los conocimientos y cuándo dejan de serlo? Y, sobre todo, icómo mediar entre los actores para llegar a un acuerdo en el que todos sientan que ganan? Estas diferencias en el discurso bien pueden estar ligadas a la falta de acuerdo epistemológico o a la predominancia de ciertos postulados sociotécnicos en 
los programas gubernamentales como, por ejemplo, el identificar que el problema de la producción de maíz en Los Altos se puede resolver dando semilla de maíz sin comprender que para los campesinos es importante conservar su semilla.

Lo relevante de la innovación, desde nuestra perspectiva, es su origen: si es propia o es ajena, quién la controla y cómo se la apropian los actores a quienes está dirigida. Coincidimos así con la propuesta de Bonfil (1988) en torno a los conceptos de cultura propia, cultura apropiada e impuesta. Los campesinos van tomando aquellas innovaciones que consideran útiles a su problemática, por lo que la identificación de los problemas no puede provenir de una imposición unilateral o que beneficie sólo a un grupo de actores, tal como se intentó con el PMS original y como se intentó llevar a cabo en 2013 con el Programa de Maíz Sustentable.

Las posiciones de los actores sociotécnicos están en su mayoría en contra del paradigma tecnológico institucional de reconversión productiva y de paquetes tecnológicos, lo cual plantea nuevas preguntas: ihasta dónde los actores implicados en una red sociotécnica pueden realmente transformar el paradigma tecnológico implícito en sus programas? La pregunta es pertinente dado que el trasfondo de las controversias planteadas refiere a la interacción "humano-planta" en la que —en opinión de los actores y de nosotros - los campesinos seguirán siendo los agentes centrales del proceso evolutivo y de selección del maíz.

\section{Notas}

${ }^{1}$ No existe un consenso en cuanto al número de variedades de maíces nativos en México. Por ejemplo, se reportan: 25 por Wellhausen y John (1952), 31 por Bretting y Goodman (1989), 59 son las más comúnmente mencionadas por Sánchez et al. (2000), 75 por Mera (2009) y Boege (2008) y 237 por Espinosa (2006).

${ }^{2}$ En Chiapas, desde hace aproximadamente sesenta años se realizan colectas, como lo documentan los trabajos del biólogo norteamericano Wellhausen et al. (1952), cuyo objetivo, además de taxonómico, es alimentar una colección ex situ para resguardar la riqueza filogenética de cada microclima y región. Las colectas de variedades realizadas desde entonces en varios estados -entre ellos Chiapas - constituyen parte del acervo que actualmente gestiona el Centro Internacional de Mejoramiento de Maíz y Trigo (CIMMYT), el cual funciona con fondos de la Fundación Rockefeller, y recientemente con apoyo de las fundaciones de Carlos Slim y Bill Gates, además de contar con apoyos del gobierno mexicano.

${ }^{3}$ CIMMYT, la Universidad Autónoma de Chapingo, el INIFAP, el CINVESTAV-Irapuato, la Facultad de Química de la UNAM, el Colegio de Posgraduados y el Instituto de Biotecnología de la UNAM (Arellano y Ortega, 2005).

${ }^{4}$ Decidimos utilizar el enfoque de soberanía alimentaria en el sentido del derecho de las comunidades campesinas a alimentarse de acuerdo con sus valores y tradiciones, con base en los recursos locales que genere la autonomía en su sentido más amplio.

${ }^{5}$ La región de Los Altos está principalmente conformada por montañas escarpadas de bosques mesófilos de pino y encino. Se ubica al noreste de la capital del estado, a una altitud promedio de 2500 metros. Aunque tiene diversos climas, en los municipios mencionados el clima es templado subhúmedo, con un promedio de temperatura entre $-3^{\circ} \mathrm{C}$ y $18^{\circ} \mathrm{C}$, y con precipitaciones anuales de entre 200 a $1800 \mathrm{~mm}$ (CONABIO).

${ }^{6}$ La milpa es un sistema de cultivos asociados principalmente maíz, frijol, calabaza y chile, pero puede incluir varias docenas de otros cultivos alimentarios y medicinales-, que permite la supervivencia biológica (Warman, 1985) y la reproducción social de los campesinos (Nigh, 1978), donde se manifiesta la diversidad biológica, la identidad, la cosmogonía y el arraigo a un territorio. La agricultura de milpa es considerada como improductiva y rústica por el paradigma tecnológico-industrial actual.

${ }^{7}$ La noción de región de Los Altos proviene de la geografía política; principalmente remite a montañas con altitudes mayores de 1400 metros sobre el nivel del mar. En este trabajo se propone la noción de región de Los Altos propuesta por Viqueira en su artículo "Los Altos 
de Chiapas: una introducción general" (2002), la cual incluye veinticinco municipios que conforman la franja mediana del macizo central —desde Zinacantán, San Cristóbal, Teopisca y Amatenango, hasta sus límites con Tabasco- - Es también descrita como un área cultural que posee una historia común de varios siglos atrás, con un constante intercambio cultural y comercial entre los hablantes de lenguas mayenses - tsotsil, tseltal, chol, las más importantes en número de hablantes-..

8 Alianza para el Campo -antes PROGRESA-, MasAgro, Programa Especial de Seguridad Alimentaria en Chiapas, Comité Sistema Producto Maíz, Programa de Apoyos para Maíz y Frijol (PROMAF); Programa de Maíces Criollos de la CONANP y Programa de Agricultura Protegida.

9 La marginación alimentaria responde a una forma de exclusión que hace más vulnerables los sistemas alimentarios en los que vive el campesino.

${ }^{10}$ Según la fuente entrevistada del polo académico, el consejo debía administrar un presupuesto de dieciséis millones de pesos de un total de 72 millones.

${ }^{11}$ Existen divergencias en torno al momento en el que se lanza públicamente el PMS. Por un lado, algunas de las organizaciones refieren el lanzamiento en febrero de 2007, mientras que otras lo ubican a finales de 2006. Esta información pone en evidencia la manipulación del programa con fines electorales de campaña electoral de la gubernatura de Chiapas en 2006.

12 Entrevista con uno de los actores institucionales involucrados en la ejecución del programa.

${ }^{13}$ Especies arvenses como los quelites (Chenopodiumsp.), verdolaga (Portulacaoleracea) y la hierba mora (Solanumsp.), cuyo consumo está fuertemente ligado al patrón de la dieta mesoamericana.

${ }^{14}$ La semilla QPM fue desarrollada por el INIFAPOcozocuautla con semilla-base del Centro Internacional de Mejoramiento de Maíz y de Trigo (CIMMYT). Se sabe que esta semilla tiene un gen opaco responsable de un alto contenido de proteína; sin embargo, dicho gen es recesivo, por lo que al cruzarse por medio de polinización libre con otras variedades tiende a perder esta característica. Más allá de las resistencias culturales que la propuesta suponía, también implicaba dificultades de tipo técnico. Al segundo año de producción de la semilla QPM, los rendimientos bajaron drásticamente y los campesinos tenían que adquirir la semilla nuevamente.

15 Actualmente Secretaría de Pueblos y Culturas Indígenas (SEPCI).

${ }^{16}$ Entrevista con uno de los participantes de la Red de Comida Sana y Cercana - polo mercado- Cabe mencionar que, además de maíz, esta red buscaba trigo, hortalizas, leche y otros productos para la conformación de un mercado orgánico en la ciudad de San Cristóbal de Las Casas.

${ }^{17}$ Esta información está basada en la entrevista a un actor del sector asociativo - polo organizacional - con una fuerte injerencia en las acciones de la red.

18 En el año 2003, la SAGARPA había lanzado el denominado Programa Tecnológico del Cultivo de Maíz Orgánico, cuyo objetivo era "promover la conservación de semillas nativas mediante paquetes tecnológicos orgánicos y la formación de productores". Dicho programa era operado por el Colegio de Posgraduados (COLPOS), institución que coordinaba las acciones con algunas organizaciones que trabajaban en municipios de Los Altos.

${ }^{19}$ Se solicitó formalmente al IFAI (oficio de solicitud núm. 0000800071613) que la SAGARPA en Tuxtla Gutiérrez nos diera acceso a las minutas de las reuniones sostenidas. En diciembre de 2013 SAGARPA en Chiapas todavía no nos había respondido.

${ }^{20}$ Para más información, consultar D’Alessandro (2013).

${ }^{21}$ También conocida como sociología del actor red, ha tenido una gran repercusión en la sociología francesa, al grado de establecer un laboratorio de investigación sobre la innovación, la ciencia y los procesos sociales cuyas figuras principales en Francia son Latour, Callon y Akrich.

${ }^{22}$ Para ver un trabajo sobre implementaciones científicas en las instituciones de desarrollo tecnológico en torno al maíz, ver Arellano (2005).

${ }^{23}$ El primer panel de diálogo "Conservación de maíces criollos" se realizó en octubre de 2011 con el objetivo de contribuir a la comprensión de los procesos políticotécnico-productivos implícitos en el discurso y acción 
de los actores ligados a la conservación de maíz criollo. Participaron investigadores especializados en el maíz en Chiapas de diferentes instituciones, como CIESAS; ECOSUR e INIFAP; representantes de organizaciones que trabajan en la producción de maíz; productores de los municipios de Tenejapa, Amatenango del Valle, Teopisca y de la región Frailesca; actores del sector público SEMARNAT-CONANP, entre otros.

${ }^{24}$ Un ejemplo reciente de esto es la venta de cuatro toneladas - de una variedad mejorada-, realizada por una sociedad anónima relacionada con el Colegio de Agrónomos de Chiapas, utilizando infraestructura y recursos humanos del INIFAP. Para más información, ver D'Alessandro (2013).

${ }^{25}$ Extensionismo se refiere a los servicios de capacitación y atención técnica específicamente para la agricultura.

26 También conocidas como despachos, conformados generalmente por profesionales especializados en la asesoría técnica agronómica y como gestores de recursos gubernamentales o de otro tipo. Ver González y Nigh (2005).

${ }^{27}$ Las organizaciones académicas referidas son El Colegio de la Frontera Sur (ECOSUR), CIESAS, Universidad Autónoma de Chiapas (UNACH), Universidad Intercultural de Chiapas (UNICH), Universidad Autónoma Chapingo (UACH), Programa de Investigaciones Multidisciplinarias sobre Mesoamérica y el Sureste-Universidad Nacional Autónoma de México (PROIMMSE-UNAM ), entre otras.

${ }^{28}$ Esta información fue descrita por uno de los actores que participó directamente en el Comité Distrital de Desarrollo Rural Sustentable de los Altos - polo institucional一.

${ }^{29}$ La codificación y análisis de las relaciones fueron realizados con el apoyo de Fredy Jiménez, estudiante de maestría en desarrollo local de la Universidad Nacional de San Martin, Buenos Aires.

${ }^{30}$ En el caso de semillas para horticultura, en el año 2005, Seminis -empresa que tiene una importante planta productora en el municipio de La Trinitaria, Chiapasse convirtió en subsidiaria exclusiva de Monsanto. Cuenta con más de 3500 patentes de semillas utilizadas en la agricultura tropical, de alto rendimiento. En el caso del maíz, de las 1049 variedades de maíz registradas ante el SNICS, cerca de novecientas pertenecen a empresas privadas, siendo las principales Monsanto y Pioneer.

\section{Referencias bibliográficas}

Aguirre Gómez, José Alfonso, (2011) Custodios de razas criollas de maíz en México. Programas de Recursos Genéticos INIFAP-CIRCE-CEBAJ. Presentación interna del INIFAP y del SINAREFI. México, SNICS-SAGARPA. Arellano Arturo y Claudia Ortega, (2005) "Las redes sociotécnicas en torno a la investigación biotecnológica del maíz" en Convergencia. Vol. 12, núm. 38 , pp. 255-276.

Bellon Mauricio R. y Eduard Taylor, (1993) "Folk Soil Taxonomy and the Partial Adoption of New Seed Varieties", en Economic Development and Cultural Change. Vol. 4l, núm. 4, pp. 763-786.

Bellon, Mauricio R, y Jon Hellin, (2011) "Planting Hybrids, Keeping Landraces: Agricultural Modernization and Tradition Among Small-Scale Maize Farmers in Chiapas, Mexico" en World Development. Vol. 39, núm. 8, pp-1434-1443.

Bellon, Mauricio R. y Brush Sthepen, (1994) "Keepers of Maize in Chiapas, Mexico” en Economic Botany. Núm. 48(2), pp. 196-209.

Bentz, Frederic, (1997) "Diversidad y distribución prehispánica del maíz mexicano" en Arqueología Mexicana. Vol. 5, núm. 25, pp. 16-23.

Boege, Eckart, (2009) "Centros de origen, pueblos indígenas y diversificación del maíz” en Ciencias. Núm. 92-93. Octubre-marzo 2009, pp. 18-28.

Boege, Eckart, (2010) Territorios y diversidad biológica. La agrobiodiversidad de los pueblos indígenas de México. México, CEDRSSA.

Bonfil, Guillermo, (1988) "La teoría del control cultural en el estudio de procesos étnicos”, Anuario Antropológico. Número 86, pp: 13-53. Disponible en: http://ciesas.edu.mx/Publicaciones/Clasicos/ articulos/TeoriadelControl.pdf [consultado el 4 de agosto de 2013].

Bretting, P.K. y M. M. Goodman, (1989) "Karyotypic Variation in Mesoamerican Races of Maize and its 
Systematic Significance" en Economic Botany. Núm. 43 , pp. 107-124.

Callon, Michel, (1986) "Some Elements of a Sociology of Translation: Domestication of the Scallope and the Fishermen of St Brieuc Bay" en J. Law (ed.), Power. Action and Belief: A New Sociology of Knowledge?, Londres, R.K.P.

Callon, Michel, (2006a) "Pour une sociologie des controverses technologiques" en Akrich, M., M. Callon y B. Latour (eds.), Sociologie de la traduction: Textes fondateurs. París, Presses de l'Ecole des Mines.

Callon, Michel, (2006b), "Sociologie de l'acteur réseau" en Akrich, M., M. Callon y B. Latour, Sociologie de la traduction: Textes fondateurs. París, Presses de l'École des Mines.

Callon, Michel; P. Laredo y P. Mustar, (1995) "Strategic Management of Research and Technology Programme Evaluation" en F. Pichault (coord.), La gestion strategique de la recherche et de la technologie: L'evaluation des programmes. París, Ecole des Mines de Paris.

D’Alessandro, Renzo, (2013) "Maíz sustentable ipara quién?" en ALAI. América Latina en Movimiento [en linea]. 25 de julio 2013, Agencia Latinoamericana de información. Disponible en http://alainet.org/ active/65957 [consultado el 12 de agosto de 2013].

Dosi, G., (1988) "The Nature of the Innovative Process" en Soete L. (ed.), Technical Change and Economic Theory. Londres, Pinter Publishers.

Eakin, H., H. Perales; K. Appendini y S. Sweeney, (en prensa) "Selling Maice in México: The Persistence of Peasant Farming in an Era of Global Markets" en Development and Change.

Espinosa, Alejandro, (2006) "Texto de comentarios al Plan Maestro para la siembra de transgénicos de maíz para SENASICA [mimeo].

González, Alma Amalia y Ronald Nigh, (2005) "Smallholder Participation and Certification of Organic Farm Products in Mexico" en Journal of Rural Studies. Vol. 21, pp. 449-460.

Groombridge, B. y M. D. Jenkins, (2002) World Atlas of Biodiversity. Earth's Living Resources in the 2lst Century, Berkeley, California; Londres, University of California Press.
Gómez Martínez, Emanuel, (2012)“Mujeres y hombres de maíz... y frijol" [documento interno de la organización Chol-Xumulha].

Hellin, Jon, Styske Groenewald y Alder Keleman (2012), "Impact pathways of trade liberalization on rural livelihoods: A case study of smallholder maize farmers in Mexico" en Iberoamerican Journal of Development Studies. Vol. 1, núm. 1. Marzo 2012. Disponible en: http://ried.unizar.es/index.php/revista/article/ view/38 [consultado el 12 de agosto de 2013].

Hernández-Xolocotzi, Efraín y A. Flores, (1971) “Estudio morfológico de cinco nuevas razas de maíz en la Sierra Madre Occidental de México: implicaciones filogenéticas y fitogeográficas" en Agrociencia. Núm. 5, pp. 3-30.

Keleman, A., Hellin, J. y M. R.Bellon, (2009) "Maize Diversity, Rural Development Policy, and Farmers' Practices: Lessons from Chiapas, Mexico" en The Geographical Journal. Vol. 175, núm. 1. Marzo 2009, pp. 52-70.

Köler, Ulric, (1975) Cambio cultural dirigido en Los Altos de Chiapas. México, INE.

Latour, Bruno y Michel Callon, (1985) "Les paradoxes de la modernité. Comment concevoir les innovations?" en Prospective et Santé. Núm. 36, pp. 13-25.

Latour, Bruno, (2005) Reassembling the Social: an Introduction to Actor-Network-Theory. Nueva York, Oxford University Press.

Mariaca, Ramón, A. González Jácome y T. Lerner, (2007) "El huerto familiar en México: avances y propuestas" en López-J. F. Olguín, A. Aragón G. y A.M. Tapia R. (eds.), Avances en agroecología y ambiente. Vol. I, pp. 119 138.

Mariaca, Ramón, Alba González Jácome y Luis Manuel Arias Reyes, (2010) El huerto maya yucateco en el siglo XVI. México, ECOSUR/CIEAM/CNCyT-Yucatán/ FMICT-Yucatán, pp. 10-23.

Mayo, Samuel y Renzo D’Alessandro, (2012) “Posponen votación sobre transgénicos” en Milenio Semanal 28 de mayo de 2012 [en línea]. Disponible en http:// www.milenio.com/cdb/doc/noticias2011/0ld491b95 ldc5336af0388c308d229c9 [consultado el 5 de julio de 2013]. 
Mera, L.M., (2009) "Diversificación y distribución reciente del maíz en México", en T.A. Kato, C. Mapes, L.M. Mera, J.A. Serratos, R.A. y Bye (eds.), Origen y diversificación del maíz: una revisión analítica. México: Universidad Nacional Autónoma de México/ Comisión Nacional para el Conocimiento y Uso de la Biodiversidad, pp. 69-81.

Montaigne, Etienne, (1997) "Théorie évolutionniste, dynamiquetechnologiqueetsystèmed'approvisionnement alimentaire". Documento redactado para el programa de la FAO Approvisionnement et distribution alimentaires des villes de l'Afrique francophone. Roma.

Parra, Manuel, T. Alemán Santillán y González A., (1989) El subdesarrollo agrícola en Los Altos de Chiapas. Texcoco, México, Universidad de Chapingo, pp. 2l-80.

Perales, Hugo, (2009) "Maíz, riqueza de México" en Ciencias. Núm. 92-93, pp. 46-55.

Perales, Hugo, Bruce Benz y Brush Stephen B., (2005) "Maize Diversity and Ethnolinguistic Eiversity in Chiapas, Mexico" en Proceedings of the National Academy of Sciences of the United States of America. Vol. 102, núm. 3, pp: 949-954.

Perales, Hugo y Juan Manuel Hernández-Casillas, (2005) "Diversidad del maíz en Chiapas" en M. González
Espinosa, N. Ramírez Marcial y L. Ruiz Montoya (eds.), Diversidad biológica de Chiapas. México, Plaza y Valdés/ECOSUR/COCYTECH.

Perales, Hugo, Brush, Stephen y C.O. Qualset, (2003) "Landraces of Maize in Central Mexico: An Altitudinal Transect" en Economic Botany. Vol. 57, número l, pp. 7-20.

Sánchez G., M.M. Goodman y C.W. Stuber, (2000) "Isozymatic and Morphological Diversity in the Races of Maize in Mexico" en Economic Botany. Número 54, pp. 43-59.

Trébuil, G. y S. P. Kam, (2000) "From Cropping and Farming Systems Research to Ecoregional Approaches for Integrated Natural Resources Management: Developing the Agricultural System Concept", ponencia presentada en Agricultural Systems for Sustainable Resources Management and Community Organization Development Proceedings of the First Thailand National Agricultural Systems Seminar. Bangkok, 16 y 17 de noviembre de 2000.

Wellhausen, Edwin John, (1952) Races of Maize in Mexico: Their Origin, Characteristics and Distribution. Estados Unidos, Harvard University. 